\title{
Pacific
}

Journal of

Mathematics

\section{STRONG SOLUTIONS TO THE COMPRESSIBLE LIQUID CRYSTAL SYSTEM}

Yu-Ming Chu, Xian-Gao LiU and Xiao LiU 


\title{
STRONG SOLUTIONS TO THE COMPRESSIBLE LIQUID CRYSTAL SYSTEM
}

\author{
Yu-Ming Chu, Xian-Gao LiU and Xiao LiU
}

\section{We prove the existence of local strong solutions of the compressible liquid crystal system.}

\section{Introduction}

We consider the following simplified system of Ericksen-Leslie equations:

$$
\begin{aligned}
\rho_{t}+\operatorname{div}(\rho u) & =0, \\
\rho u_{t}+\rho u \cdot \nabla u+\nabla p-\mu \Delta u+\lambda\left(\operatorname{div}(\nabla n \otimes \nabla n)-\nabla \frac{|\nabla n|^{2}}{2}\right) & =0, \\
\frac{\partial n}{\partial t}+u \cdot \nabla n-v\left(\Delta n+|\nabla n|^{2} n\right) & =0,
\end{aligned}
$$

with the following initial and boundary conditions:

$$
\begin{gathered}
\left.(\rho, u, n)\right|_{t=0}=\left(\rho_{0}, u_{0}, n_{0}\right), \quad x \in \Omega, \\
u(x, t)=u_{0}(x)=0, \quad n(x, t)=n_{0}(x), \quad x \in \partial \Omega,
\end{gathered}
$$

where $u$ is the velocity field, $n$ the macroscopic average of the nematic liquid crystal orientation field, $\rho_{0} \geq 0,\left|n_{0}\right|=1$, and pressure $p=a \rho^{\gamma}$ with $\gamma>1$, where $\gamma$ is the adiabatic constant (in the physically relevant case of a monoatomic gas, $\gamma=\frac{5}{3}$ ). This system is modeled after the theory of Oseen [1933] and Frank [1958]; see the articles [Ericksen 1962; Forster et al. 1971; Leslie 1966; 1968] or the books [Ericksen and Kinderlehrer 1987; Gennes and Prost 1993; Pasechnik et al. 2009; Stephen 1970; Xie 1988].

The system (1.1)-(1.3) is much more complicated than the compressible NavierStokes equations, because equation (1.3), like the situation with heat flow into a sphere, makes the strongly coupling term $\operatorname{div}(\nabla n \otimes \nabla n)-\nabla \frac{|\nabla n|^{2}}{2}$ have a weak convergence. So far, the existence of weak solutions to the system remains open, though there are celebrated contributions by Lions [1998]; see also [Feireisl 2004;

This work was supported partly by NSFC grant 11071043,11131005 , and 11071069 .

MSC2010: 76N10, 35Q35, 35Q30.

Keywords: strong solutions, compressible liquid crystals, local existence. 
Feireisl et al. 2001]. Liu and Qing [2011] proved the global existence of finite energy weak solutions to the case where the free energy is replaced by the GinzburgLandau approximation energy,

$$
\min _{n \in H^{1}\left(\Omega ; R^{3}\right)} \int_{\Omega} \frac{1}{2}|\nabla n|^{2}+\frac{1}{4 \sigma^{2}}\left(|n|^{2}-1\right)^{2} d x .
$$

In the incompressible case, F. H. Lin and C. Liu, among others [Lin 1989; Lin and Liu 1995; Lin and Liu 2001; Lin and Liu 2000; Lin and Liu 1996; Calderer and Liu 2000], systematically studied the incompressible liquid crystal dynamics system based on the Ericksen-Leslie model (that is, the Ginzburg-Landau approximation case with $\rho$ being a constant in system (1.1) makes the velocity field divergence free) and proved the global existence of weak solutions, classical solutions, and partial regularity. Liu and Zhang [2009] also studied the existence of weak solutions to the incompressible liquid crystal system with the Ginzburg-Landau approximation and $\rho$ nonconstant.

It is well known that there exist no global solutions to the system (1.1)-(1.3) even in the incompressible case. Surprisingly, we can prove the local existence of a strong solution to the compressible liquid crystal system with initial density $\rho_{0} \geq 0$. We gained enlightenment from the corresponding results of the compressible Navier-Stokes equations. There is a huge literature on the compressible Navier-Stokes equations, under the crucial assumption that the initial density $\rho_{0}$ is bounded below away from zero. The existence results were obtained by Nash, Itaya, Tani, Matsumura, and Nishida, among others. For general nonnegative initial density, Cho, Kim, and Choe [Choe and Kim 2003; Cho et al. 2004; Cho and Kim 2006] obtained the existence of a local strong solution to a compressible NavierStokes equation.

We first have the energy law

$$
\frac{d E}{d t}+\int_{\Omega} \mu|\nabla u|^{2}+\left.\left.\lambda v|\Delta n+| \nabla n\right|^{2} n\right|^{2}=0
$$

with

$$
E(t)=\int_{\Omega}\left(\frac{1}{2} \rho u^{2}+\frac{\lambda}{2}|\nabla n|^{2}+\frac{a}{\gamma-1} \rho^{\gamma}\right) .
$$

From the definition of velocity,

$$
\begin{aligned}
\frac{d x(X, t)}{d t} & =u(x(X, t), t), \\
x(X, 0) & =X .
\end{aligned}
$$

The continuity equation can be rewritten as

$$
\frac{d \rho(x(X, t), t)}{d t}+\rho \operatorname{div} u=0,
$$


that is,

$$
\rho(x, t)=\rho_{0} \exp \left(-\int_{0}^{t} \operatorname{div} u\right) .
$$

We need the following regularity for $\rho_{0}, n_{0}$, and $u_{0}$ :

$$
\rho_{0} \in W^{1,6}(\Omega), \quad u_{0} \in H_{0}^{1}(\Omega) \cap H^{2}(\Omega), \quad n_{0} \in H^{3}(\Omega) .
$$

We also need some compatibility condition on the initial data: for some $g \in L^{2}$,

$$
\mu \Delta u_{0}-\lambda \operatorname{div}\left(\nabla n_{0} \otimes \nabla n_{0}-\frac{1}{2}\left|\nabla n_{0}\right|^{2} I\right)-a \nabla \rho_{0}^{\gamma}=\rho_{0}^{\frac{1}{2}} g .
$$

The following is our main result.

Theorem 1.1. Assume $\Omega$ is a smooth bounded domain in $\mathbb{R}^{3}$ and $\left(\rho_{0}, n_{0}, u_{0}\right)$ satisfies regularity condition (1.9) and compatibility condition (1.10). Then there exist a small time $T^{*}>0$ and a unique strong solution $(\rho, n, u)$ of the compressible liquid crystal system (1.1)-(1.3) in $\left(0, T^{*}\right) \times \Omega$, satisfying initial and boundary conditions (1.4) and (1.5), such that

$$
\begin{aligned}
\rho & \in C\left(\left[0, T^{*}\right) ; W^{1,6}\right), & & \rho_{t} \in C\left(\left[0, T^{*}\right) ; L^{6}\right), \\
u & \in C\left(\left[0, T^{*}\right) ; H_{0}^{1} \cap H^{2}\right) \cap L^{2}\left(0, T^{*} ; W^{2,6}\right), & & u_{t} \in L^{2}\left(0, T^{*} ; H_{0}^{1}\right), \\
n & \in C\left(\left[0, T^{*}\right) ; H^{2}\right) \cap L^{2}\left(0, T^{*} ; W^{2,6}\right), & & n_{t} \in C\left(\left[0, T^{*}\right) ; H_{0}^{1}\right), \\
\sqrt{\rho} u_{t} & \in C\left(\left[0, T^{*}\right) ; L^{2}\right) . & &
\end{aligned}
$$

\section{Approximation solutions}

We now consider the linearized equations as follows: for fixed smooth functions $v, d: \Omega \times[0, T] \rightarrow \mathbb{R}^{3}$ with

$$
\frac{d x(X, t)}{d t}=v(x(X, t), t)
$$

and $x(X, 0)=X$, and $v(x, 0)=u_{0}(x), d(x, 0)=n_{0}(x)$,

$$
\rho_{t}+\operatorname{div}(\rho v)=0
$$

$$
\begin{gathered}
(\rho u)_{t}+\operatorname{div}(\rho v \otimes v)+a \nabla \rho^{\gamma}=\mu \Delta u-\lambda \operatorname{div}\left(\nabla n \otimes \nabla n-\frac{1}{2}|\nabla n|^{2} I\right), \\
n_{t}-\gamma \Delta n=\lambda|\nabla d|^{2} d-v \cdot \nabla d,
\end{gathered}
$$

with initial and boundary conditions

$$
\begin{gathered}
\left.(\rho, u, n)\right|_{t=0}=\left(\rho_{0}+\delta, u_{0}, n_{0}\right), \quad x \in \Omega, \\
u(x, t)=u_{0}(x)=0, \quad n(x, t)=n_{0}(x), \quad x \in \partial \Omega .
\end{gathered}
$$

Here $\delta>0$ is a constant, and $\rho_{0} \geq 0,\left|n_{0}\right|=1$. 
We use the following notations: Suppose Banach spaces

$$
\begin{aligned}
& \mathscr{A}=L^{\infty}\left(0, T ; H^{2}(\Omega)\right) \cap L^{2}\left(0, T ; W^{2,6}(\Omega)\right) \cap W_{2}^{1,1}((0, T) \times \Omega), \\
& \mathscr{B}=L^{\infty}\left(0, T ; W^{2,6}(\Omega)\right) \cap W_{\infty}^{1,1}((0, T) \times \Omega) \cap W_{2}^{2,1}((0, T) \times \Omega)
\end{aligned}
$$

with norm respectively

$$
\begin{aligned}
& \|v\|_{\mathscr{A}}=\|v\|_{L^{\infty}\left(0, T ; H^{2}(\Omega)\right)}+\|v\|_{L^{2}\left(0, T ; W^{2,6}(\Omega)\right)}+\left\|v_{t}\right\|_{L^{2}\left(0, T ; H^{1}(\Omega)\right)}, \\
& \|d\|_{\mathscr{B}}=\left\|d_{t}\right\|_{L^{2}\left(0, T ; H^{2}(\Omega)\right)}+\left\|d_{t}\right\|_{L^{\infty}\left(0, T ; H^{1}(\Omega)\right)}+\|d\|_{L^{\infty}\left(0, T ; W^{2,6}(\Omega)\right)} .
\end{aligned}
$$

Lemma 2.1. For given $v$ with $\|v\|_{\mathscr{A}} \leq A$, the unique solution $\rho$ of (2.1) satisfies

$$
\begin{aligned}
\|\rho\|_{L^{\infty}\left(0, T ; W^{1,6}(\Omega)\right)} & \leq c c_{0}\left(1+T^{\frac{1}{2}} A\right) \exp \left(c T^{\frac{1}{2}} A\right), \\
\left\|\rho_{t}\right\|_{L^{\infty}\left(0, T ; L^{6}(\Omega)\right)} & \leq c c_{0} A \exp \left(c T^{\frac{1}{2}} A\right) .
\end{aligned}
$$

In particular,

$$
\begin{aligned}
&\|p\|_{L^{\infty}\left(0, T ; W^{1,6}(\Omega)\right)} \leq c c_{0}\left(1+T^{\frac{1}{2}} A\right) \exp \left(c T^{\frac{1}{2}} A\right), \\
&\left\|p_{t}\right\|_{L^{\infty}\left(0, T ; L^{6}(\Omega)\right)} \leq c c_{0} A \exp \left(c T^{\frac{1}{2}} A\right),
\end{aligned}
$$

where $c$ is an absolute constant, perhaps dependent on $\Omega, \lambda, \mu, \gamma$, etc., and $c_{0}$ is a constant dependent on initial and boundary data.

Proof. Since

$$
\begin{aligned}
\nabla \rho & =\nabla \rho_{0} \exp \left(-\int_{0}^{t} \operatorname{div} v\right)-\rho_{0} \int_{0}^{t} \nabla \operatorname{div} v \exp \left(-\int_{0}^{t} \operatorname{div} v\right), \\
\rho_{t} & =-\rho_{0} \operatorname{div} v \exp \left(-\int_{0}^{t} \operatorname{div} v\right),
\end{aligned}
$$

we have, from the Minkowski inequality,

$$
\begin{aligned}
\|\nabla \rho\|_{L^{6}(\Omega)} & \leq c\left\|\rho_{0}\right\|_{W^{1,6}(\Omega)}\left(1+\left\|\int_{0}^{t} \nabla^{2} v\right\|_{L^{6}(\Omega)}\right) \exp \left(\int_{0}^{T}\|\operatorname{div} v\|_{L^{\infty}(\Omega)}\right) \\
& \leq c\left\|\rho_{0}\right\|_{W^{1,6}(\Omega)}\left(1+\int_{0}^{T}\left\|\nabla^{2} v\right\|_{L^{6}(\Omega)}\right) \exp \left(\int_{0}^{T}\|\operatorname{div} v\|_{L^{\infty}(\Omega)}\right) \\
& \leq c\left\|\rho_{0}\right\|_{W^{1,6}(\Omega)}\left(1+T^{\frac{1}{2}}\|v\|_{X}\right) \exp \left(c T^{\frac{1}{2}}\|v\|_{X}\right) \\
& \leq c c_{0}\left(1+T^{\frac{1}{2}} A\right) \exp \left(c T^{\frac{1}{2}} A\right), \\
\left\|\rho_{t}\right\|_{L^{6}(\Omega)} & \leq c\left\|\rho_{0}\right\|_{L^{\infty}(\Omega)}\|\nabla v\|_{L^{6}(\Omega)} \exp \left(\int_{0}^{T}\|\operatorname{div} v\|_{L^{\infty}(\Omega)}\right) \\
& \leq c c_{0} \exp \left(c T^{\frac{1}{2}} A\right)\|v\|_{H^{2}(\Omega)} \leq c c_{0} A \exp \left(c T^{\frac{1}{2}} A\right),
\end{aligned}
$$

where $X=L^{2}\left(0, T ; W^{2,6}(\Omega)\right)$. 
Lemma 2.2. Suppose $\|v\|_{\mathscr{A}} \leq A,\|d\|_{\Re} \leq B$. Then (2.3) with initial condition $n(x, 0)=n_{0}(x)$ has a unique solution $n$ and a constant $K_{1}$, depending only on $n_{0}$ and $u_{0}$, such that, for $T=T(A, B)$ small enough,

(2.10) $\|n\|_{\mathscr{B}}=\left\|n_{t}\right\|_{L^{2}\left(0, T ; H^{2}(\Omega)\right)}+\left\|n_{t}\right\|_{L^{\infty}\left(0, T ; H^{1}(\Omega)\right)}+\|n\|_{L^{\infty}\left(0, T ; W^{2,6}(\Omega)\right)} \leq K_{1}$.

Proof. The existence of a solution to (2.3) is standard. We just give the estimates as follows. Differentiating (2.3) with respect to time $t$,

$$
n_{t t}-v \Delta n_{t}=v\left(|\nabla d|_{t}^{2} d+|\nabla d|^{2} d_{t}\right)+\left(v_{t} \cdot \nabla\right) d-(v \cdot \nabla) d_{t} .
$$

Multiplying by $\Delta n_{t}$, integrating over $\Omega$, and using the Cauchy inequality, we get

$$
\begin{aligned}
\frac{1}{2} \frac{d}{d t} & \int_{\Omega}\left|\nabla n_{t}\right|^{2}+v \int_{\Omega}\left|\Delta n_{t}\right|^{2} \\
& =-\int_{\Omega} v\left(|\nabla d|_{t}^{2} d+|\nabla d|^{2} d_{t}\right) \cdot \Delta n_{t}+\left(v_{t} \cdot \nabla\right) d \cdot \Delta n_{t}-(v \cdot \nabla) d_{t} \cdot \Delta n_{t} \\
& \leq \int_{\Omega} 2 v|\nabla d|\left|\nabla d_{t}\right||d|\left|\Delta n_{t}\right|+v|\nabla d|^{2}\left|d_{t}\right|\left|\Delta n_{t}\right| \\
& \quad+\int_{\Omega}\left|\nabla v_{t}\right||\nabla d|\left|\nabla n_{t}\right|+\left|v_{t}\right|\left|\nabla^{2} d\right|\left|\nabla n_{t}\right|+|v|\left|\nabla d_{t}\right|\left|\Delta n_{t}\right| \\
& =\sum_{i=1}^{5} I_{i} .
\end{aligned}
$$

We have the following estimates for $I_{i}$ :

$$
\begin{aligned}
I_{1} & =\int_{\Omega} 2 v|\nabla d|\left|\nabla d_{t}\right||d|\left|\Delta n_{t}\right| \leq c \int_{\Omega}|\nabla d|^{2}\left|\nabla d_{t}\right|^{2}|d|^{2}+\frac{v}{6}\left\|\Delta n_{t}\right\|_{L^{2}(\Omega)}^{2}, \\
I_{2} & =\int_{\Omega} v|\nabla d|^{2}\left|d_{t}\right|\left|\Delta n_{t}\right| \leq c \int_{\Omega}|\nabla d|^{4}\left|d_{t}\right|^{2}+\frac{v}{6}\left\|\Delta n_{t}\right\|_{L^{2}}^{2}, \\
I_{3} & =\int_{\Omega}\left|\nabla v_{t}\right||\nabla d|\left|\nabla n_{t}\right| \leq A^{-2} B^{-2} \int_{\Omega}\left|\nabla v_{t}\right|^{2}|\nabla d|^{2}+A^{2} B^{2} \int_{\Omega}\left|\nabla n_{t}\right|^{2}, \\
I_{4} & =\int_{\Omega}\left|v_{t}\right|\left|\nabla^{2} d\right|\left|\nabla n_{t}\right| \leq A^{-2} B^{-2} \int_{\Omega}\left|v_{t}\right|^{2}\left|\nabla^{2} d\right|^{2}+A^{2} B^{2} \int_{\Omega}\left|\nabla n_{t}\right|^{2} \\
& \leq c A^{-2} B^{-2}\left\|\nabla v_{t}\right\|_{L^{2}}^{2}\left\|\nabla^{2} d\right\|_{L^{2}}\left\|\nabla^{2} d\right\|_{L^{6}}+A^{2} B^{2} \int_{\Omega}\left|\nabla n_{t}\right|^{2}, \\
I_{5} & =\int_{\Omega}|v|\left|\nabla d_{t}\right|\left|\Delta n_{t}\right| \leq \frac{3}{v} \int_{\Omega}|v|^{2}\left|\nabla d_{t}\right|^{2}+\frac{v}{6}\left\|\Delta n_{t}\right\|_{L^{2}}^{2} .
\end{aligned}
$$

Substituting all the estimates into (2.11), we get

$$
\begin{aligned}
\frac{d}{d t} \int_{\Omega}\left|\nabla n_{t}\right|^{2}+v \int_{\Omega}\left|\Delta n_{t}\right|^{2} \leq\left. c \int_{\Omega}|\nabla d|^{2}\left|\nabla d_{t}\right|^{2}|| d\right|^{2}+c \int_{\Omega}|\nabla d|^{4}\left|d_{t}\right|^{2} \\
+c A^{-2} B^{-2} \int_{\Omega}\left|\nabla v_{t}\right|^{2}|\nabla d|^{2}+c A^{2} B^{2} \int_{\Omega}\left|\nabla n_{t}\right|^{2} \\
+c \int_{\Omega}|v|^{2}\left|\nabla d_{t}\right|^{2}+c A^{-2} B^{-2}\left\|\nabla v_{t}\right\|_{L^{2}}^{2}\left\|\nabla^{2} d\right\|_{L^{2}}\left\|\nabla^{2} d\right\|_{L^{6}},
\end{aligned}
$$


that is,

$$
\begin{aligned}
& \int_{\Omega}\left|\nabla n_{t}\right|^{2}+v \int_{0}^{T} \int_{\Omega}\left|\Delta n_{t}\right|^{2} \\
& \quad \leq c B^{6} T+c A^{2} B^{2} T+c+c A^{2} B^{2} \int_{0}^{T} \int_{\Omega}\left|\nabla n_{t}\right|^{2}+c\left(n_{0}, u_{0}\right),
\end{aligned}
$$

where

$c\left(n_{0}, u_{0}\right)$

$$
=c \int_{\Omega}\left|\Delta \nabla n_{0}\right|^{2}+\left|\nabla n_{0}\right|^{2}\left|\nabla^{2} n_{0}\right|^{2}+\left|\nabla n_{0}\right|^{6}+c \int_{\Omega}\left|\nabla u_{0}\right|^{2}\left|\nabla n_{0}\right|^{2}+\left|u_{0}\right|^{2}\left|\nabla^{2} n_{0}\right|^{2} .
$$

Using Gronwall's inequality, we obtain

$$
\int_{\Omega}\left|\nabla n_{t}\right|^{2} \leq\left(c B^{6} T+c A^{2} B^{2} T+c_{0}\right) \exp \left(c A^{2} B^{2} T\right)
$$

and

$$
\int_{\Omega}\left|\nabla n_{t}\right|^{2}+v \int_{0}^{T} \int_{\Omega}\left|\Delta n_{t}\right|^{2} \leq c\left(B^{6} T+A^{2} B^{2} T+c_{0}\right)\left(1+\exp \left(c A^{2} B^{2} T\right)\right) .
$$

Taking $T=T(A, B)$ small, we get

$$
\int_{\Omega}\left|\nabla n_{t}\right|^{2}+v \int_{0}^{T} \int_{\Omega}\left|\Delta n_{t}\right|^{2} \leq c .
$$

The elliptic estimates can be deduced from (2.3):

$$
\begin{aligned}
\|n\|_{W^{2,6}(\Omega)} & \leq\left\|n_{t}\right\|_{L^{6}}+\|v \cdot \nabla d\|_{L^{6}}+\left\||\nabla d|^{2} d\right\|_{L^{6}}+\left\|n_{0}\right\|_{W^{2,6}} \\
& \leq\|v \cdot \nabla d\|_{L^{6}}+\left\||\nabla d|^{2} d\right\|_{L^{6}}+c_{0}
\end{aligned}
$$

We estimate each item:

$\|v \cdot \nabla d\|_{L^{6}}$

$$
\begin{aligned}
& =\left(\int_{\Omega}|v|^{6}|\nabla d|^{6}\right)^{\frac{1}{6}} \leq\left(\int_{\Omega}\left|v-u_{0}\right|^{6}|\nabla d|^{6}\right)^{\frac{1}{6}}+\left\|u_{0}\right\|_{L^{\infty}}\left(\int_{\Omega}|\nabla d|^{6}\right)^{\frac{1}{6}} \\
& \leq c B\left(\int_{\Omega}\left|\nabla v-\nabla u_{0}\right|^{2}\right)^{\frac{1}{2}}+c\left\|u_{0}\right\|_{L^{\infty}}\left(\int_{\Omega}\left|\nabla d-\nabla n_{0}\right|^{6}\right)^{\frac{1}{6}}+c\left\|u_{0}\right\|_{L^{\infty}}\left\|\nabla n_{0}\right\|_{L^{\infty}} \\
& \leq c B\left(\int_{\Omega}\left|\int_{0}^{t} \nabla v_{t}\right|^{2}\right)^{\frac{1}{2}}+c_{0} B^{\frac{2}{3}}\left(\int_{\Omega}\left|\int_{0}^{t} \nabla d_{t}\right|^{2}\right)^{\frac{1}{6}}+c_{0} \\
& \leq c B T^{\frac{1}{2}}\left\|\nabla v_{t}\right\|_{L^{2}\left(Q_{T}\right)}+c_{0} T^{\frac{1}{3}} B+c_{0} \leq c A B T^{\frac{1}{2}}+c_{0} B T^{\frac{1}{3}}+c_{0}
\end{aligned}
$$


and

$$
\begin{aligned}
\left\||\nabla d|^{2} d\right\|_{L^{6}} & =\left(\left.\left.\int_{\Omega}|| \nabla d\right|^{2} d\right|^{6}\right)^{\frac{1}{6}} \leq\left(\int_{\Omega}|\nabla d|^{12}\left|d-n_{0}\right|^{6}\right)^{\frac{1}{6}}+c_{0}\left(\int_{\Omega}|\nabla d|^{12}\right)^{\frac{1}{6}} \\
& \leq c B^{2}\left(\int_{\Omega}\left|d-n_{0}\right|^{6}\right)^{\frac{1}{6}}+c_{0}\left(\int_{\Omega}\left|\nabla d-\nabla n_{0}\right|^{12}\right)^{\frac{1}{6}}+c_{0} \\
& \leq c B^{2}\left(\int_{\Omega}\left|\nabla d-\nabla n_{0}\right|^{2}\right)^{\frac{1}{2}}+c_{0} B\left(\int_{\Omega}\left|\nabla d-\nabla n_{0}\right|^{6}\right)^{\frac{1}{6}}+c_{0} \\
& \leq c A B^{2} T^{\frac{1}{2}}+c_{0} B^{2} T^{\frac{1}{3}}+c_{0} .
\end{aligned}
$$

Taking $T=T(A, B)$ small enough, we obtain the desired $\|n\|_{W^{2,6}} \leq c_{0}$.

For (2.2) we have following Lemma.

Lemma 2.3. Under the conditions of Lemma 2.2, suppose $n$ satisfies (2.3) and $\rho$ (2.1). Then there exists a unique solution u satisfying (2.2), and there is a constant $K_{2}$, depending only on $n_{0}$ and $u_{0}$, such that, for $T=T(A, B)$ small enough,

(2.12) $\|u\|_{\mathscr{A}} \equiv\|u\|_{L^{\infty}\left(0, T ; H^{2}(\Omega)\right)}+\|u\|_{L^{2}\left(0, T ; W^{2,6}(\Omega)\right)}+\left\|u_{t}\right\|_{L^{2}\left(0, T ; H^{1}(\Omega)\right)} \leq K_{2}$.

Proof. Since

$$
\rho \geq \delta \exp \left(-\int_{0}^{T}|\nabla v|_{L^{\infty}((0, T) \times \Omega)}\right)>0,
$$

the standard theory of parabolic equations implies the existence of the solution to (2.2). Differentiating (2.2) with respect to time $t$, we get

(2.13) $\rho u_{t t}-\mu \Delta u_{t}$

$=-\lambda \operatorname{div}\left((\nabla d \otimes \nabla d)_{t}-\frac{1}{2}|\nabla d|_{t}^{2} I\right)-\nabla p_{t}-(\rho v \cdot \nabla) v_{t}-\left(\rho_{t} v \cdot \nabla\right) v-\left(\rho v_{t} \cdot \nabla\right) v-\rho_{t} u_{t}$.

Multiplying by $u_{t}$, integrating by parts, and using the continuity of (2.1), we get

$$
\begin{aligned}
& \frac{1}{2} \frac{d}{d t} \int_{\Omega} \rho\left|u_{t}\right|^{2}+\mu \int_{\Omega}\left|\nabla u_{t}\right|^{2} \\
& =\lambda \int_{\Omega}\left((\nabla d \otimes \nabla d)_{t}-\frac{1}{2}|\nabla d|_{t}^{2} I\right) \cdot \nabla u_{t} \\
& -\int_{\Omega} \nabla p_{t} \cdot u_{t}-(\rho v \cdot \nabla) v_{t} \cdot u_{t}-\left(\rho_{t} v \cdot \nabla\right) v \cdot u_{t}-\int_{\Omega}\left(\rho v_{t} \cdot \nabla\right) v \cdot u_{t}+\rho_{t}\left|u_{t}\right|^{2} \\
& \leq 3 \lambda \int_{\Omega}|\nabla d|\left|\nabla d_{t}\right|\left|\nabla u_{t}\right|+\int_{\Omega} p_{t} \operatorname{div}\left(u_{t}\right)+\rho|v|\left|\nabla v_{t}\right|\left|u_{t}\right| \\
& +\int_{\Omega} \rho|v||\nabla v|^{2}\left|u_{t}\right|+\rho|v|^{2}\left|\nabla^{2} v\right|\left|u_{t}\right|+\rho|v||\nabla v|\left|\nabla u_{t}\right| \\
& +\int_{\Omega} \rho\left|v_{t}\right||\nabla v|\left|u_{t}\right|+2 \rho|v|\left|\nabla u_{t}\right|\left|u_{t}\right| \\
& =\sum_{i=1}^{8} I_{i}
\end{aligned}
$$


For each $I_{i}$ we have

$$
\begin{aligned}
I_{1} & =3 \lambda \int_{\Omega}|\nabla d|\left|\nabla d_{t}\right|\left|\nabla u_{t}\right| \leq c \int_{\Omega}|\nabla d|^{2}\left|\nabla d_{t}\right|^{2}+\frac{\mu}{12} \int_{\Omega}\left|\nabla u_{t}\right|^{2} \leq c B^{4}+\frac{\mu}{12} \int_{\Omega}\left|\nabla u_{t}\right|^{2}, \\
I_{2} & =\int_{\Omega} p_{t} \operatorname{div}\left(u_{t}\right) \leq c \int_{\Omega}\left|p_{t}\right|^{2}+\frac{\mu}{12} \int_{\Omega}\left|\nabla u_{t}\right|^{2} \\
& \leq c_{0} \exp \left(\int_{0}^{T} 2\|\nabla v\|_{L^{\infty}(\Omega)}\right) \int_{\Omega}|\nabla v|^{2}+\frac{\mu}{12} \int_{\Omega}\left|\nabla u_{t}\right|^{2} \\
& \leq c_{0} A^{2} \exp \left(c A T^{\frac{1}{2}}\right)+\frac{\mu}{12} \int_{\Omega}\left|\nabla u_{t}\right|^{2}, \\
I_{3} & =\int_{\Omega}|\rho||v|\left|\nabla v_{t}\right|\left|u_{t}\right| \leq A^{4} \int_{\Omega} \rho\left|u_{t}\right|^{2}+c_{0} A^{-2} \exp \left(c A T^{\frac{1}{2}}\right) \int_{\Omega}\left|\nabla v_{t}\right|^{2}, \\
I_{4} & =\int_{\Omega}|\rho||v||\nabla v|^{2}\left|u_{t}\right| \leq A^{6} \int_{\Omega} \rho\left|u_{t}\right|^{2}+c_{0} \exp \left(c A T^{\frac{1}{2}}\right), \\
I_{5} & =\int_{\Omega}|\rho||v|^{2}\left|\nabla^{2} v\right|\left|u_{t}\right| \leq A^{6} \int_{\Omega} \rho\left|u_{t}\right|^{2}+c_{0} \exp \left(c A T^{\frac{1}{2}}\right), \\
I_{6} & =\int_{\Omega} \rho|v||\nabla v|\left|\nabla u_{t}\right| \leq c \int_{\Omega} \rho^{2}|v|^{2}|\nabla v|^{2}+\frac{\mu}{12} \int_{\Omega}\left|\nabla u_{t}\right|^{2} \\
& \leq c_{0} A^{4} \exp \left(c A T^{\frac{1}{2}}\right)+\frac{\mu}{12} \int_{\Omega}\left|\nabla u_{t}\right|^{2}, \\
I_{7} & =\int_{\Omega} \rho\left|v_{t}\right||\nabla v|\left|u_{t}\right| \leq A^{4} \int_{\Omega} \rho\left|u_{t}\right|^{2}+A^{-4} \int_{\Omega} \rho\left|v_{t}\right|^{2}|\nabla v|^{2} \\
& \leq A^{2} \int_{\Omega} \rho\left|u_{t}\right|^{2}+c_{0} A^{-2} \exp \left(c A T^{\frac{1}{2}}\right) \int_{\Omega}\left|v_{t}\right|^{2}, \\
I_{8} & =2 \int_{\Omega} \rho|v|\left|\nabla u_{t}\right|\left|u_{t}\right| \leq c \int_{\Omega} \rho\left|u_{t}\right|^{2}\left(\rho|v|^{2}\right)+\frac{\mu}{12} \int_{\Omega}\left|\nabla u_{t}\right|^{2} \\
& \leq c_{0} A^{2} \exp \left(c A T^{\frac{1}{2}}\right) \int_{\Omega} \rho\left|u_{t}\right|^{2}+\frac{\mu}{12} \int_{\Omega}\left|\nabla u_{t}\right|^{2} .
\end{aligned}
$$

From the above estimates, we get

$$
\begin{aligned}
\int_{\Omega} \rho\left|u_{t}\right|^{2}+ & \int_{0}^{T} \int_{\Omega}\left|\nabla u_{t}\right|^{2} \\
& \leq c B^{4} T+c_{0} A^{4} T \exp \left(c A T^{\frac{1}{2}}\right)+c_{0}+c_{0} A^{4} \exp \left(c A T^{\frac{1}{2}}\right) \int_{0}^{T} \int_{\Omega} \rho\left|u_{t}\right|^{2},
\end{aligned}
$$

which implies that

$$
\int_{\Omega} \rho\left|u_{t}\right|^{2}+\int_{0}^{T} \int_{\Omega}\left|\nabla u_{t}\right|^{2} \leq\left(c B^{4} T+c_{0} A^{4} T \exp \left(c A T^{\frac{1}{2}}\right)\right) c_{0} A^{4} T \exp \left(c A T^{\frac{1}{2}}\right) .
$$


Taking $T=T(A, B)$ small enough, we deduce

$$
\int_{\Omega} \rho\left|u_{t}\right|^{2}+\int_{0}^{T} \int_{\Omega}\left|\nabla u_{t}\right|^{2} \leq C\left(c_{0}\right) .
$$

Finally, we estimate

$$
\|u\|_{L^{\infty}\left(0, T ; H^{2}(\Omega)\right)} \quad \text { and } \quad\|u\|_{L^{2}\left(0, T ; W^{2,6}(\Omega)\right)} .
$$

From (2.2), we get

$\|u\|_{H^{2}(\Omega)}$

$$
\leq c\left(\|\nabla p\|_{L^{2}(\Omega)}+\left\|\rho u_{t}\right\|_{L^{2}(\Omega)}+\left\|\nabla^{2} n \nabla n\right\|_{L^{2}(\Omega)}\right)+c\left(\|(\rho v \cdot \nabla) v\|_{L^{2}(\Omega)}+c_{0}\right) .
$$

Now we have

$$
\begin{aligned}
\|\nabla p\|_{L^{2}(\Omega)} & \leq c_{0} \exp \left(c A T^{\frac{1}{2}}\right)+c_{0} A T^{\frac{1}{2}} \exp \left(c A T^{\frac{1}{2}}\right), \\
\left\|\rho u_{t}\right\|_{L^{2}(\Omega)} & \leq c_{0} \exp \left(c A T^{\frac{1}{2}}\right)\left\|\sqrt{\rho} u_{t}\right\|_{L^{2}(\Omega)}, \\
\left\|\nabla^{2} n \nabla n\right\|_{L^{2}(\Omega)} & \leq\left\|\nabla^{2} n\right\|_{L^{6}(\Omega)}\|\nabla n\|_{L^{2}(\Omega)}^{\frac{1}{2}}\|\nabla n\|_{L^{6}(\Omega)}^{\frac{1}{2}} \leq K_{1}^{2},
\end{aligned}
$$

and

$\|\rho v \cdot \nabla v\|_{L^{2}(\Omega)}^{2}$

$$
\begin{aligned}
& \leq\|\rho\|_{L^{\infty}(\Omega)}^{2} \int_{\Omega}|v|^{2}|\nabla v|^{2} \\
& \leq c_{0} \exp \left(c A T^{\frac{1}{2}}\right)\left(\int_{\Omega}\left|v-u_{0}\right|^{2}|\nabla v|^{2}+\left\|u_{0}\right\|_{L^{\infty}}^{2} \int_{\Omega}\left|\nabla v-\nabla u_{0}\right|^{2}+c_{0}\right) \\
& \leq c_{0} \exp \left(c A T^{\frac{1}{2}}\right)\left(\int_{\Omega}\left|\int_{0}^{t} v_{t}\right|^{2}|\nabla v|^{2}+c_{0} \int_{\Omega}\left|\int_{0}^{t} \nabla v_{t}\right|^{2}+c_{0}\right) \\
& \leq c_{0} \exp \left(c A T^{\frac{1}{2}}\right)\left(A^{4} T+c_{0} A^{2} T+c_{0}\right) .
\end{aligned}
$$

Similarly, we have

$$
\begin{aligned}
\|\nabla p\|_{L^{6}(\Omega)} & \leq c_{0} \exp \left(c A T^{\frac{1}{2}}\right)+c_{0} A T^{\frac{1}{2}} \exp \left(c A T^{\frac{1}{2}}\right), \\
\left\|\rho u_{t}\right\|_{L^{2}\left(0, T ; L^{6}(\Omega)\right)} & \leq c_{0} \exp \left(c A T^{\frac{1}{2}}\right)\left\|\nabla u_{t}\right\|_{L^{2}\left(0, T ; L^{2}(\Omega)\right)} \\
& \leq c_{0} \exp \left(c A T^{\frac{1}{2}}\right) C\left(c_{0}\right), \\
\left\|\nabla^{2} n \nabla n\right\|_{L^{2}\left(0, T ; L^{6}(\Omega)\right)} & \leq\left\|\nabla^{2} n\right\|_{L^{2}\left(0, T ; L^{6}(\Omega)\right)}\|\nabla n\|_{L^{\infty}(\Omega)} \leq K_{1}^{2},
\end{aligned}
$$


and

$$
\begin{aligned}
\|\rho v \cdot \nabla v\|_{L^{2}\left(0, T ; L^{6}(\Omega)\right)}^{2} & \leq\|\rho\|_{L^{\infty}(\Omega)}^{2} \int_{0}^{T}\left(\int_{\Omega}|v|^{6}|\nabla v|^{6}\right)^{\frac{1}{3}} \\
& \leq c_{0} \exp \left(c A T^{\frac{1}{2}}\right) \int_{0}^{T}\|v\|_{L^{\infty}(\Omega)}^{2}\|\nabla v\|_{L^{\infty}(\Omega)}^{\frac{4}{3}} \times\left(\int_{\Omega}\left|\nabla v-\nabla u_{0}\right|^{2}+1\right)^{\frac{1}{3}} \\
& \leq c_{0} \exp \left(c A T^{\frac{1}{2}}\right) A^{2} \int_{0}^{T}\|\nabla v\|_{L^{\infty}(\Omega)}^{\frac{4}{3}} \times\left(\int_{\Omega}\left|\int_{0}^{t} \nabla v_{t}\right|^{2}+1\right)^{\frac{1}{3}} \\
& \leq c_{0} \exp \left(c A T^{\frac{1}{2}}\right)\left(T \int_{0}^{T} \int_{\Omega}\left|\nabla v_{t}\right|^{2}+1\right)^{\frac{1}{3}} \times\left(\int_{0}^{T}\|v\|_{W^{2,6}(\Omega)}^{2}\right)^{\frac{2}{3}} T^{\frac{1}{3}} \\
& \leq c_{0} \exp \left(c A T^{\frac{1}{2}}\right)\left(T A^{2}+1\right)^{\frac{1}{3}} A^{\frac{4}{3}} T^{\frac{1}{3}} .
\end{aligned}
$$

Thus

$\int_{\Omega} \rho\left|u_{t}\right|^{2} d x+\mu \int_{0}^{T} \int_{\Omega}\left|\nabla u_{t}\right|^{2} d x d t+\|u\|_{L^{\infty}\left(0, T ; H^{2}(\Omega)\right)}+\|u\|_{L^{2}\left(0, T ; W^{2,6}(\Omega)\right)} \leq C\left(c_{0}\right)$.

This concludes the proof.

If $\left(n^{\delta}, u^{\delta}\right)$ denotes a unique solution of (2.2) and (2.3) with

$$
\rho(x, 0)=\rho_{0}+\delta
$$

and initial and boundary conditions, then taking $\delta \rightarrow 0$, we obtain a unique solution $(n, u)$ of the linearized system $(2.1)-(2.3)$ with $\rho(x, 0)=\rho_{0}$ and initial and boundary conditions such that $\|n\|_{\mathscr{B}} \leq K_{1},\|u\|_{\mathscr{A}} \leq K_{2}$. So we can define a map

$$
\mathcal{T}: \mathcal{W} \rightarrow \mathcal{W}, \quad(d, v) \mapsto(n, u),
$$

where Banach space

$$
\mathscr{W}=(\mathscr{A} \otimes \mathscr{B}) \cap \mathscr{C}=\mathscr{A} \otimes \mathscr{B}
$$

with

$$
\mathscr{C}=\left\{(n, u):\|(n, u)\|_{\mathscr{C}}=\|n\|_{L^{2}\left(0, T ; H^{2}(\Omega)\right)}+\|u\|_{L^{2}\left(0, T ; H^{1}(\Omega)\right)}<\infty\right\} .
$$

The following lemma tells us that the map $\mathscr{T}$ is contracted in the sense of weaker norm for $(d, v) \in \mathscr{W}$.

Lemma 2.4. There is a constant $0<\theta<1$ such that for any $\left(d^{i}, v^{i}\right) \in \mathcal{W}, i=1,2$,

$$
\left\|\mathscr{T}\left(d^{1}, v^{1}\right)-\mathscr{T}\left(d^{2}, v^{2}\right)\right\|_{\mathscr{C}} \leq \theta\left\|\left(d^{1}-d^{2}, v^{1}-v^{2}\right)\right\|_{\mathscr{C}}
$$

for some small $T>0$. 
Proof. Suppose $\rho_{i}, n^{i}$, and $u^{i}$ are the solutions to (2.1)-(2.3) corresponding to given $\left(d^{i}, v^{i}\right) \in \mathcal{W}$. Define $\rho=\rho_{2}-\rho_{1}, d=d^{2}-d^{1}, v=v^{2}-v^{1}, n=n^{2}-n^{1}$, $u=u^{2}-u^{1}$, and

$$
\rho_{i}=\rho_{0} \exp \left(-\int_{0}^{t} \operatorname{div} v^{i}\right)
$$

$i=1,2$. Then

$$
\begin{aligned}
& \rho_{t}+\operatorname{div}\left(\rho v^{2}\right)=-\operatorname{div}\left(\rho_{1} v\right), \\
& n_{t}-v \Delta n=v\left|\nabla d^{2}\right|^{2} d^{2}-v\left|\nabla d^{1}\right|^{2} d^{1}-v^{2} \nabla d^{2}+v^{1} \nabla d^{1}, \\
& \rho_{2} u_{t}-\mu \Delta u=\left(\rho_{1}-\rho_{2}\right) u_{t}^{1}+\rho_{1} v^{1} \nabla v^{1}-\rho_{2} v^{2} \nabla v^{2}+\nabla p_{1} \\
& \quad-\nabla p_{2}-\lambda \nabla \cdot\left(\nabla n^{2} \otimes \nabla n^{2}-\frac{1}{2}\left|\nabla n^{2}\right|^{2} I\right) \\
& \quad+\lambda \nabla \cdot\left(\nabla n^{1} \otimes \nabla n^{1}-\frac{1}{2}\left|\nabla n^{1}\right|^{2} I\right) .
\end{aligned}
$$

Multiplying (2.16) by $n$ and integrating over $\Omega$, we get

$$
\begin{aligned}
\frac{1}{2} \frac{d}{d t} \int_{\Omega}|n|^{2} d x+ & v \int_{\Omega}|\nabla n|^{2} d x \\
& \leq \int_{\Omega}\left|\nabla d^{2}\right|^{2} d^{2} \cdot n-\left|\nabla d^{1}\right|^{2} d^{1} \cdot n-v \nabla d^{2} \cdot n-v^{1} \nabla d \cdot n \\
& \leq \eta \int_{\Omega}\left(|\nabla d|^{2}+|\nabla v|^{2}\right)+c(\eta, A, B) \int_{\Omega}|n|^{2},
\end{aligned}
$$

where $c(\eta, A, B)(s)$ satisfies

$$
\int_{0}^{T} c(\eta, A, B)(s) d s \leq K_{3}
$$

for small $T=T(A, B, \eta)$, where $K_{3}$ is a constant dependent on initial and boundary data $c_{0}$.

Differentiating (2.16) with respect to $x_{i}$, multiplying by $\nabla n$, and integrating over $\Omega$, we deduce

$$
\begin{aligned}
\frac{1}{2} \frac{d}{d t} \int_{\Omega}|\nabla n|^{2} d x & +\frac{v}{2} \int_{\Omega}\left|\nabla^{2} n\right|^{2} d x \\
& \leq \eta \int_{\Omega}\left(|\nabla v|^{2}+|\nabla d|^{2}+\left|\nabla^{2} d\right|^{2}\right)+c(\eta, A, B) \int_{\Omega}|\nabla n|^{2},
\end{aligned}
$$

where $c(\eta, A, B)$ satisfies (2.19), and we have used the following identities and estimates:

$$
\begin{aligned}
\nabla d^{2} \nabla^{2} d^{2} d^{2}-\nabla d^{1} \nabla^{2} d^{1} d^{1} & =\nabla d \nabla^{2} d^{2} d^{1}+\nabla d^{1} \nabla^{2} d d^{1}+\nabla d^{1} \nabla^{2} d^{1} d, \\
\left|\nabla d^{2}\right|^{2} \nabla d^{2}-\left|\nabla d^{1}\right|^{2} \nabla d^{1} & =\left|\nabla d^{2}\right|^{2} \nabla d+\left(\left|\nabla d^{2}\right|^{2}-\left|\nabla d^{1}\right|^{2}\right) \nabla d^{1},
\end{aligned}
$$


and

$$
\begin{aligned}
\int_{\Omega}|\nabla n|^{2}\left|\nabla^{2} d^{2}\right|^{2} & \leq\left(\int_{\Omega}\left|\nabla^{2} d^{2}\right|^{6}\right)^{\frac{1}{3}}\left(\int_{\Omega}|\nabla n|^{3}\right)^{\frac{2}{3}} \\
& \leq c B^{2}\|\nabla n\|_{L^{2}(\Omega)}\left\|\nabla^{2} n\right\|_{L^{2}(\Omega)} \leq \frac{v}{2} \int_{\Omega}\left|\nabla^{2} n\right|^{2}+c B^{4} \int_{\Omega}|\nabla n|^{2} .
\end{aligned}
$$

Multiplying (2.15) by $\rho$ and using the Minkowski inequality, we have

$$
\begin{aligned}
\frac{d}{d t} \int_{\Omega} \frac{1}{2}|\rho|^{2} & =\int_{\Omega}-\frac{1}{2}|\rho|^{2} \operatorname{div} v^{2}-\int_{\Omega} \rho\left(\nabla \rho_{1} v+\rho_{1} \operatorname{div} v\right) \\
& \leq c \int_{\Omega}|\rho|^{2}\left|\nabla v^{2}\right|+c\|\rho\|_{L^{2}(\Omega)}\left\|\nabla \rho_{1}\right\|_{L^{3}(\Omega)}\|v\|_{L^{6}(\Omega)} \\
& \quad+c\|\rho\|_{L^{2}(\Omega)}\left\|\rho_{1}\right\|_{L^{\infty}(\Omega)}\|\nabla v\|_{L^{2}(\Omega)} \\
& \leq c\left\|v^{2}\right\|_{W^{2,6}(\Omega)}\|\rho\|_{L^{2}(\Omega)}^{2}+\eta\|\nabla v\|_{L^{2}(\Omega)}^{2} \quad+c_{0} \eta^{-1} \exp \left(c A T^{\frac{1}{2}}\right)\left(1+\left\|\int_{0}^{t} \nabla^{2} v^{1}\right\|_{L^{3}(\Omega)}^{2}\right)\|\rho\|_{L^{2}(\Omega)}^{2} \\
& \leq \eta\|\nabla v\|_{L^{2}(\Omega)}^{2}+c\left\|v^{2}\right\|_{W^{2,6}(\Omega)}\|\rho\|_{L^{2}(\Omega)}^{2} \\
& +c_{0} \eta^{-1} \exp \left(c A T^{\frac{1}{2}}\right)\left(1+T\left\|\nabla^{2} v^{1}\right\|_{L^{2}\left(0, T ; L^{6}(\Omega)\right.}^{2}\right)\|\rho\|_{L^{2}(\Omega)}^{2} \\
& \leq c_{0} \eta^{-1} \exp \left(c A T^{\frac{1}{2}}\right)\left(1+T A^{2}+\left\|v^{2}\right\|_{W^{2,6}(\Omega)}\right)\|\rho\|_{L^{2}(\Omega)}^{2}+\eta\|\nabla v\|_{L^{2}(\Omega)}^{2},
\end{aligned}
$$

that is,

$$
\frac{d}{d t} \int_{\Omega} \frac{1}{2}|\rho|^{2} \leq \eta\|\nabla v\|_{L^{2}(\Omega)}^{2}+c(\eta, A, T)\|\rho\|_{L^{2}(\Omega)}^{2},
$$

where $c(\eta, A, T)$ satisfies (2.19).

Multiplying (2.17) by $u$ and integrating over $\Omega$, we deduce

$$
\begin{aligned}
& \text { (2.22) } \frac{1}{2} \frac{d}{d t} \int_{\Omega} \rho_{2}|u|^{2} d x+\mu \int_{\Omega}|\nabla u|^{2} d x \\
& =\int_{\Omega}-\rho_{2} v^{2} u \nabla u+\left(\rho_{1}-\rho_{2}\right) u_{t}^{1} \cdot u+\rho_{1} v^{1} \nabla v^{1} \cdot u-\rho_{2} v^{2} \nabla v^{2} \cdot u+\left(p_{2}-p_{1}\right) \operatorname{div} u
\end{aligned}
$$
$+\lambda\left(\nabla n^{2} \otimes \nabla n^{2}-\frac{1}{2}\left|\nabla n^{2}\right|^{2} I\right) \nabla u-\lambda\left(\nabla n^{1} \otimes \nabla n^{1}-\frac{1}{2}\left|\nabla n^{1}\right|^{2} I\right) \nabla u$

$=\int_{\Omega}-\rho_{2} v^{2} u \nabla u+\left(\rho_{1}-\rho_{2}\right)\left(u_{t}^{1}+v^{1} \nabla v^{1}\right) \cdot u$

$-\rho_{2}\left(v \nabla v^{2}+v^{1} \nabla v\right) \cdot u+\left(p_{1}-p_{2}\right) \operatorname{div} u$

$$
+\lambda\left(\nabla n^{2} \otimes \nabla n^{2}-\frac{1}{2}\left|\nabla n^{2}\right|^{2} I\right) \nabla u-\lambda\left(\nabla n^{1} \otimes \nabla n^{1}-\frac{1}{2}\left|\nabla n^{1}\right|^{2} I\right) \nabla u
$$

$\leq \eta \int_{\Omega}|\nabla v|^{2}+\frac{2 \mu}{3} \int_{\Omega}|\nabla u|^{2}+c(\eta, A, B) \int_{\Omega} \rho_{2}|u|^{2}+|\rho|^{2}+|\nabla n|^{2}$, 
where $c(\eta, A, B)$ satisfying (2.19). Here we have used the key estimates

$$
\begin{aligned}
\int_{\Omega} \rho_{2}\left|v \nabla v^{2}+v^{1} \nabla v\right||u| \leq & \left\|\nabla v^{2}\right\|_{L^{6}(\Omega)}\left\|\rho_{2} u\right\|_{L^{2}(\Omega)}\|v\|_{L^{6}(\Omega)} \\
& +\|\nabla v\|_{L^{2}(\Omega)}\left\|\rho_{2} u\right\|_{L^{2}(\Omega)}\left\|v^{1}\right\|_{L^{\infty}(\Omega)} \\
\leq & c_{0} \exp \left(c A T^{\frac{1}{2}}\right)\left\|\sqrt{\rho_{2}} u\right\|_{L^{2}(\Omega)}\left\|\nabla v^{2}\right\|_{H^{2}(\Omega)}\|\nabla v\|_{L^{2}(\Omega)} \\
& \quad+c_{0} \exp \left(c A T^{\frac{1}{2}}\right)\left\|\sqrt{\rho_{2}} u\right\|_{L^{2}(\Omega)}\left\|v^{1}\right\|_{H^{2}(\Omega)}\|\nabla v\|_{L^{2}(\Omega)} \\
\leq & \eta\|\nabla v\|_{L^{2}(\Omega)}^{2}+c \eta^{-1} A^{2} \exp \left(c A T^{\frac{1}{2}}\right)\left\|\sqrt{\rho_{2}} u\right\|_{L^{2}(\Omega)}^{2}, \\
\int_{\Omega}|\nabla n||\nabla u|\left|\nabla n^{2}\right| \leq & \eta \int_{\Omega}|\nabla u|^{2}+c \eta^{-1}\left|\nabla n^{2}\right|_{L^{\infty}(\Omega)}^{2} \int_{\Omega}|\nabla n|^{2} \\
\leq & \frac{\mu}{3} \int_{\Omega}|\nabla u|^{2}+c B^{2} \int_{\Omega}|\nabla n|^{2},
\end{aligned}
$$

and

$$
\begin{aligned}
\int_{\Omega}\left(\rho_{1}-\rho_{2}\right)\left(u_{t}^{1}+v^{1} \nabla v^{1}\right) \cdot u & \leq\|\rho\|_{L^{\frac{3}{2}}(\Omega)}\left\|u_{t}^{1}+v^{1} \nabla v^{1}\right\|_{L^{6}(\Omega)}\|u\|_{L^{6}(\Omega)} \\
& \leq c\|\rho\|_{L^{2}(\Omega)}\left\|u_{t}^{1}+v^{1} \nabla v^{1}\right\|_{H^{1}(\Omega)}\|\nabla u\|_{L^{2}(\Omega)} \\
& \leq \frac{\mu}{3}\|\nabla u\|_{L^{2}(\Omega)}^{2}+c(A, T)(t)\|\rho\|_{L^{2}(\Omega)}^{2},
\end{aligned}
$$

where $c(\eta, A, T)(t)$ satisfies (2.19).

Summing inequalities (2.18) and (2.20)-(2.22), we obtain

$$
\begin{aligned}
& \frac{d}{d t} \int_{\Omega}|\rho|^{2}+|n|^{2}+|\nabla n|^{2}+\rho_{2}|u|^{2}+\int_{\Omega}|\nabla n|^{2}+\left|\nabla^{2} n\right|^{2}+|\nabla u|^{2} \\
& \leq c \eta \int_{\Omega}|\nabla v|^{2}+|\nabla d|^{2}+\left|\nabla^{2} d\right|^{2}+c(\eta, A, B, T) \int_{\Omega}|\rho|^{2}+|n|^{2}+|\nabla n|^{2}+\rho_{2}|u|^{2},
\end{aligned}
$$

which implies, by (2.19) and taking $T=T(\eta, A, B))$ small enough,

$$
\begin{aligned}
\int_{\Omega}|\rho|^{2}+|n|^{2} & +|\nabla n|^{2}+\rho_{2}|u|^{2} \\
& \leq \eta \exp \left(\int_{0}^{T} c(\eta, A, B)(s) d s\right) \int_{0}^{T} \int_{\Omega}|\rho|^{2}+|n|^{2}+|\nabla n|^{2}+\rho_{2}|u|^{2} \\
& \leq c \eta \int_{0}^{T} \int_{\Omega}|\rho|^{2}+|n|^{2}+|\nabla n|^{2}+\rho_{2}|u|^{2} .
\end{aligned}
$$

Thus, taking $\eta$ small, we obtain

$$
\|\rho\|_{L^{\infty}\left(0, T ; L^{2}(\Omega)\right)}+\|n\|_{L^{\infty}\left(0, T ; H^{1}(\Omega)\right)}+\left\|\sqrt{\rho_{2}} u\right\|_{L^{\infty}\left(0, T ; L^{2}(\Omega)\right)} \leq c
$$


and

$$
\int_{0}^{T} \int_{\Omega}|\nabla n|^{2}+\left|\nabla^{2} n\right|^{2}+|\nabla u|^{2} \leq \theta \int_{0}^{T} \int_{\Omega}|\nabla d|^{2}+\left|\nabla^{2} d\right|^{2}+|\nabla v|^{2}
$$

with $0<\theta<1$. Since $n$ and $u$ are zero on boundary, we finish the proof.

\section{Proof of Theorem 1.1}

Proof. By the contractibility of $\mathscr{T}$, we can easily obtain a unique solution $(n, u)$ of (1.3) and (1.2), and $\rho$ is from $u$ by formula (1.8), that is, $\rho$ is a unique solution of (1.1). Lemmas 2.1-2.3 and the lower semicontinuity of norms imply that the solutions $(\rho, n, u)$ satisfy the same estimates. Multiplying (1.3) by $n$, we get

$$
|n|_{t}^{2}+(u \cdot \nabla)|n|^{2}=v \Delta|n|^{2}+\left(|n|^{2}-1\right)|\nabla n|^{2},
$$

that is,

$$
\left(|n|^{2}-1\right)_{t}+(u \cdot \nabla)\left(|n|^{2}-1\right)=v \Delta\left(|n|^{2}-1\right)+\left(|n|^{2}-1\right)|\nabla n|^{2} .
$$

Define $D=\left(|n|^{2}-1\right) \exp \left(\|\nabla n\|_{L^{\infty}\left(Q_{T}\right)}^{2} t\right)$, where $Q_{T}=\Omega \times[0, T]$. Then

$$
D_{t}+(u \cdot \nabla) D=v \Delta D+\left(|\nabla n|^{2}-\|\nabla n\|_{L_{\left(Q_{T}\right)}^{2}}^{2} D\right.
$$

with $\left.D\right|_{\partial \Omega}=0$. So from the maximum principle of parabolic equations,we deduce

$$
D \equiv 0 \quad \text { in } \quad((0, T) \times \Omega)
$$

Thus we complete the proof of the theorem.

\section{References}

[Calderer and Liu 2000] M. C. Calderer and C. Liu, "Liquid crystal flow: dynamic and static configurations", SIAM J. Appl. Math. 60:6 (2000), 1925-1949 (electronic). MR 1763310 (2001e:76009) Zbl 0956.35104

[Cho and Kim 2006] Y. Cho and H. Kim, "Existence results for viscous polytropic fluids with vacuum", J. Differential Equations 228:2 (2006), 377-411. MR 2289539 (2007j:35155) Zbl 1135. 35071

[Cho et al. 2004] Y. Cho, H. J. Choe, and H. Kim, "Unique solvability of the initial boundary value problems for compressible viscous fluids", J. Math. Pures Appl. (9) 83:2 (2004), 243-275. MR 2038120 (2005a:76133) Zbl 1080.35066

[Choe and Kim 2003] H. J. Choe and H. Kim, "Strong solutions of the Navier-Stokes equations for isentropic compressible fluids", J. Differential Equations 190:2 (2003), 504-523. MR 1970039 (2004b:35258) Zbl 1022.35037

[Ericksen 1962] J. L. Ericksen, "Hydrostatic theory of liquid crystals", Arch. Rational Mech. Anal. 9 (1962), 371-378. MR 0137403 (25 \#855) Zbl 0105.23403 
[Ericksen and Kinderlehrer 1987] J. L. Ericksen and D. Kinderlehrer (editors), Theory and applications of liquid crystals, vol. 5, The IMA Volumes in Mathematics and its Applications, Springer, New York, 1987. Papers from the IMA workshop held in Minneapolis, Minn., January 21-25, 1985, Edited by J. L. Ericksen and D. Kinderlehrer. MR 900827 (88d:82007) Zbl 0713.76006

[Feireisl 2004] E. Feireisl, Dynamics of viscous compressible fluids, vol. 26, Oxford Lecture Series in Mathematics and its Applications, Oxford University Press, Oxford, 2004. MR 2040667 (2005i:76092) Zbl 1080.76001

[Feireisl et al. 2001] E. Feireisl, A. Novotný, and H. Petzeltová, "On the existence of globally defined weak solutions to the Navier-Stokes equations", J. Math. Fluid Mech. 3:4 (2001), 358-392. MR 1867887 (2002k:35253) Zbl 0997.35043

[Forster et al. 1971] D. Forster, T. Lubensky, P. Martin, J. Swift, and P. Pershan, "Hydrodynamics of liquid crystals”, Phys. Rev. Lett. 26:17 (1971), 1016-1019.

[Frank 1958] F. C. Frank, "On the theory of liquid crystals", Discussions Faraday Soc. 25 (1958), $19-28$.

[Gennes and Prost 1993] P. G. de Gennes and J. Prost, The physics of liquid crystals, 2nd ed., International Series of Monographs on Physics (Oxford, England) 83, Clarendon, Oxford, 1993.

[Leslie 1966] F. M. Leslie, "Some constitutive equations for anisotropic fluids", Quart. J. Mech. Appl. Math. 19 (1966), 357-370. MR 0207302 (34 \#7118) Zbl 0148.20504

[Leslie 1968] F. M. Leslie, "Some constitutive equations for liquid crystals", Arch. Rational Mech. Anal. 28:4 (1968), 265-283. MR 1553506 Zbl 0159.57101

[Lin 1989] F.-H. Lin, "Nonlinear theory of defects in nematic liquid crystals; phase transition and flow phenomena", Comm. Pure Appl. Math. 42:6 (1989), 789-814. MR 1003435 (90g:82076) Zbl 0703.35173

[Lin and Liu 1995] F.-H. Lin and C. Liu, "Nonparabolic dissipative systems modeling the flow of liquid crystals", Comm. Pure Appl. Math. 48:5 (1995), 501-537. MR 1329830 (96a:35154) Zbl 0842.35084

[Lin and Liu 1996] F.-H. Lin and C. Liu, "Partial regularity of the dynamic system modeling the flow of liquid crystals", Discrete Contin. Dynam. Systems 2:1 (1996), 1-22. MR 1367385 (96m:35255) Zb1 0948.35098

[Lin and Liu 2000] F.-H. Lin and C. Liu, "Existence of solutions for the Ericksen-Leslie system", Arch. Ration. Mech. Anal. 154:2 (2000), 135-156. MR 1784963 (2003a:76014) Zbl 0963.35158

[Lin and Liu 2001] F. Lin and C. Liu, "Static and dynamic theories of liquid crystals", J. Partial Differential Equations 14:4 (2001), 289-330. MR 1883167 (2003b:82063)

[Lions 1998] P.-L. Lions, Mathematical topics in fluid mechanics. Vol. 2, vol. 10, Oxford Lecture Series in Mathematics and its Applications, The Clarendon Press Oxford University Press, New York, 1998. Compressible models, Oxford Science Publications. MR 1637634 (99m:76001) Zbl 0908.76004

[Liu and Qing 2011] X. Liu and J. Qing, "Globally weak solutions to the flow of compressible liquid crystals system”, preprint, 2011. To appear in Discret. Contin. Dyn. Syst. A.

[Liu and Zhang 2009] X. Liu and Z. Zhang, "Global existence of weak solutions for the incompressible liquid crystals", Chinese Ann. Math. 30:1 (2009), 1-20.

[Oseen 1933] C. W. Oseen, "The theory of liquid crystals", Trans. Faraday Soc. 29 (1933), 883-899. Zbl 0008.04203

[Pasechnik et al. 2009] S. V. Pasechnik, V. G. Chigrinov, and D. V. Shmeliova, Liquid crystals: viscous and elastic properties, Wiley-VCH, Weinheim, 2009. Zbl 0999.35078 
[Stephen 1970] M. J. Stephen, "Hydrodynamics of liquid crystals", Phys. Rev. A 2:4 (1970), 15581562.

[Xie 1988] Y. Z. Xie, The physics of liquid crystals, Scientific Press, Beijing, 1988.

Received May 16, 2011. Revised November 12, 2011.

YU-Ming Chu

SCHOOL OF MATHEMATICS AND COMPUTATION SCIENCES

HUNAN CITY UNIVERSITY

YIYANG, 413000

CHINA

chuyuming@hutc.zj.cn

Xian-Gao LiU

INSTITUTE OF MATHEMATICS

FUDAN UNIVERSITY

SHANGHAI, 200433

CHINA

xgliu@ fudan.edu.cn

Xiao LiU

InSTITUTE OF MATHEMATICS

FUDAN UNIVERSITY

SHANGHAI, 200433

CHINA

shaw0820@gmail.com 


\title{
PACIFIC JOURNAL OF MATHEMATICS
}

\author{
http://pacificmath.org \\ Founded in 1951 by \\ E. F. Beckenbach (1906-1982) and F. Wolf (1904-1989)
}

\section{EDITORS}

V. S. Varadarajan (Managing Editor)

Department of Mathematics

University of California

Los Angeles, CA 90095-1555

pacific@math.ucla.edu

Vyjayanthi Chari

Department of Mathematics

University of California

Riverside, CA 92521-0135

chari@math.ucr.edu

\section{Robert Finn}

Department of Mathematics Stanford University

Stanford, CA 94305-2125

finn@math.stanford.edu

Kefeng Liu

Department of Mathematics

University of California

Los Angeles, CA 90095-1555

liu@math.ucla.edu
Darren Long

Department of Mathematics

University of California

Santa Barbara, CA 93106-3080

long@math.ucsb.edu

Jiang-Hua Lu

Department of Mathematics

The University of Hong Kong

Pokfulam Rd., Hong Kong jhlu@maths.hku.hk

Alexander Merkurjev

Department of Mathematics

University of California

Los Angeles, CA 90095-1555

merkurev@math.ucla.edu
Sorin Popa

Department of Mathematics University of California

Los Angeles, CA 90095-1555 popa@math.ucla.edu

Jie Qing

Department of Mathematics

University of California

Santa Cruz, CA 95064

qing@cats.ucsc.edu

Jonathan Rogawski

Department of Mathematics

University of California

Los Angeles, CA 90095-1555

jonr@math.ucla.edu

\section{PRODUCTION}

pacific@math.berkeley.edu

\section{SUPPORTING INSTITUTIONS}

ACADEMIA SINICA, TAIPEI

CALIFORNIA INST. OF TECHNOLOGY INST. DE MATEMÁTICA PURA E APLICADA KEIO UNIVERSITY

MATH. SCIENCES RESEARCH INSTITUTE NEW MEXICO STATE UNIV.

OREGON STATE UNIV.

\author{
STANFORD UNIVERSITY \\ UNIV. OF BRITISH COLUMBIA \\ UNIV. OF CALIFORNIA, BERKELEY \\ UNIV. OF CALIFORNIA, DAVIS \\ UNIV. OF CALIFORNIA, LOS ANGELES \\ UNIV. OF CALIFORNIA, RIVERSIDE \\ UNIV. OF CALIFORNIA, SAN DIEGO \\ UNIV. OF CALIF., SANTA BARBARA
}

\author{
UNIV. OF CALIF., SANTA CRUZ \\ UNIV. OF MONTANA \\ UNIV. OF OREGON \\ UNIV. OF SOUTHERN CALIFORNIA \\ UNIV. OF UTAH \\ UNIV. OF WASHINGTON \\ WASHINGTON STATE UNIVERSITY
}

These supporting institutions contribute to the cost of publication of this Journal, but they are not owners or publishers and have no responsibility for its contents or policies.

See inside back cover or pacificmath.org for submission instructions.

The subscription price for 2012 is US \$420/year for the electronic version, and \$485/year for print and electronic.

Subscriptions, requests for back issues from the last three years and changes of subscribers address should be sent to Pacific Journal of Mathematics, P.O. Box 4163, Berkeley, CA 94704-0163, U.S.A. Prior back issues are obtainable from Periodicals Service Company, 11 Main Street, Germantown, NY 12526-5635. The Pacific Journal of Mathematics is indexed by Mathematical Reviews, Zentralblatt MATH, PASCAL CNRS Index, Referativnyi Zhurnal, Current Mathematical Publications and the Science Citation Index.

The Pacific Journal of Mathematics (ISSN 0030-8730) at the University of California, c/o Department of Mathematics, 969 Evans Hall, Berkeley, CA 94720-3840, is published monthly except July and August. Periodical rate postage paid at Berkeley, CA 94704, and additional mailing offices. POSTMASTER: send address changes to Pacific Journal of Mathematics, P.O. Box 4163, Berkeley, CA 94704-0163.

PJM peer review and production are managed by EditFLOW ${ }^{\mathrm{TM}}$ from Mathematical Sciences Publishers.

PUBLISHED BY PACIFIC JOURNAL OF MATHEMATICS

at the University of California, Berkeley 94720-3840

A NON-PROFIT CORPORATION

Typeset in LATEX

Copyright $(02012$ by Pacific Journal of Mathematics 


\section{PACIFIC JOURNAL OF MATHEMATICS}

Volume $257 \quad$ No. $1 \quad$ May 2012

Energy and volume of vector fields on spherical domains

FABiano G. B. BRito, ANDRÉ O. Gomes and Giovanni S. Nunes

Maps on 3-manifolds given by surgery

BOLDIZSÁR KALMÁR and ANDRÁS I. STIPSICZ

Strong solutions to the compressible liquid crystal system

Yu-Ming ChU, Xian-Gao LiU and XIAO LIU

Presentations for the higher-dimensional Thompson groups $n V$

Johanna HenNig and FranCESCo MatuCCI

Resonant solutions and turning points in an elliptic problem with oscillatory

boundary conditions

ALFONSO CASTRO and ROSA PARDO

Relative measure homology and continuous bounded cohomology of topological pairs

\section{Roberto Frigerio and CRISTINA PAgLiAnTINi}

Normal enveloping algebras

ALEXANDRE N. GrishKov, Marina RASSKazova and SALVATORE SICILIANO

Bounded and unbounded capillary surfaces in a cusp domain

YASUNORI AOKI and DAVID SIEGEL

On orthogonal polynomials with respect to certain discrete Sobolev inner product

Francisco Marcellán, Ramadan Zejnullahu, Bujar Fejzullahu and EDMUNDO HUERTAS

Green versus Lempert functions: A minimal example

PASCAL THOMAS

Differential Harnack inequalities for nonlinear heat equations with potentials under the Ricci flow

JIA-YONG WU

On overtwisted, right-veering open books

PAOLO LISCA

Weakly Krull domains and the composite numerical semigroup ring $D+E\left[\Gamma^{*}\right]$

JUNG WOOK LIM

Arithmeticity of complex hyperbolic triangle groups 